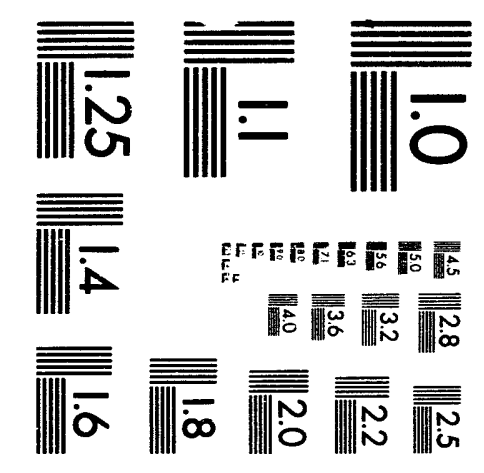


PROGRESS REPORT

DOE/ER/40412-2 73

$1 / 1 / 89-12 / 31 / 89$

GRANT * DE-FG-02-88ER40412

\section{"The Study of Hadronic Matter at the Highest Energy Density: The Ssearch for the Deconfined Quark-Gluon Phase Using 2 TEV P-P Collisions"}

\section{DISCLAIMER}

This report was prepared as an account of work sponsored by an agency of the United States Government. Neither the United States Government nor any agency thereof, nor any of their employees, makes any warranty, express or implied, or assumes any legal liability or responsibility for the accuracy, completeness, or usefulness of any information, apparatus, product, or process disclosed, or represents that its use would not infringe privately owned rights. Reference herein to any specific commercial product, process, or service by trade name, trademark, manufacturer, or otherwise does not necessarily constitute or imply its endorsement, recommendation, or favoring by the United States Government or any agency thereof. The views and opinions of authors expressed herein do not necessarily state or reflect those of the United States Government or any agency thereof.

\section{Professor Rolf Scharenberg Professor Andrew Hirsch \\ Department of Physics Purdue Research Foundation West Lafayette, IN 47907}

December 1989 


\section{OUTLINE}

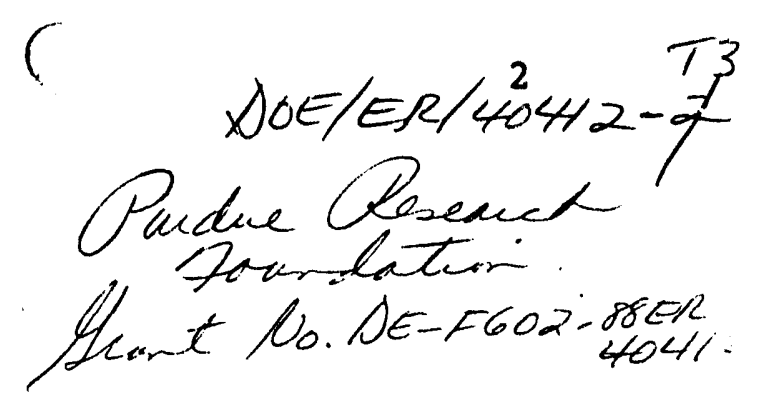

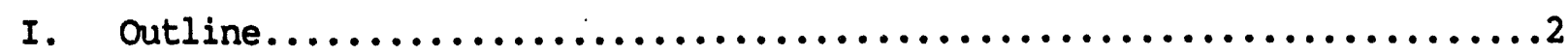

II. Running E-735, $1988-1989 \ldots \ldots \ldots \ldots \ldots \ldots \ldots \ldots \ldots \ldots \ldots \ldots \ldots \ldots$

III. Publications from the $1987-1988$ run of $E-735 \ldots \ldots \ldots \ldots \ldots \ldots \ldots \ldots$

IV. Exclusive Nuclear Fragmentation experiment at................ the Bevelac using the new TPC.

v. Research and development on parallel plate.................. avalanche detectors.

vI. Data analysis..................................

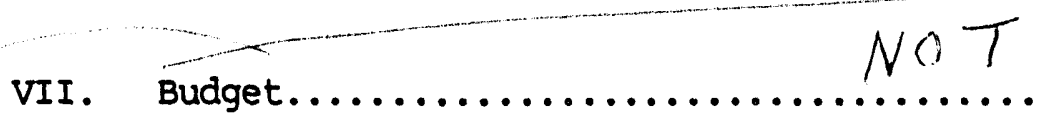

INCLUDED. de

VIII. Budget comments...................................

IX. Appended papers 
II. Running E-735 at FNAL 1988 - 1989

The Purdue group installed the central tracking chamber (CTC) at the C0 interaction region in November of 1988. Running the experiment continued around the clock until June 1989. The chamber ran successfully during this period, while exposed to $10,000 \mathrm{R}$ of radiation. Signal wires that drew too much current were reconditioned by reverse biasing. Careful attention to chamber segmentation allowed the chamber to function correctly during the whole run. Near the end of the run one section required a lower field voltage but remained operational all in all 3000 wires including $~ 600$ signal wires remained intact. Examination of the data tapes indicated that the chamber was capable of it's design goal; the identification of $\sim 80$ tracks $(\mathrm{NC})$ in the central rapidity region (Fig. 1). Some 5000 data tapes were acquired in this run. The major task of simulation and data analysis is proceeding (see VI).

III. Publications

The papers discussed in this section are based on the 1987 test run. The lower energy data was obtained during the latter stages of the 1988-1989 production run. The 40 fold increase in statistics where $\sqrt{\mathrm{s}}=1.8 \mathrm{TeV}$ will benefit the study of the highest multiplicity events i.e. $d_{N} / d y>15$.

a) The average transverse momentum $\langle P t \geq$ as a function of charged particle multiplicity. The data from our Phys. Rev. Lett. 60, 1622, 1988 is shown in Fig. 2. The flattening of $\left\langle\mathrm{P}_{t}\right\rangle$ with increasing $\mathrm{dN}_{c} / d y$ is evident. If $\left\langle P_{t}\right\rangle$ should rise for $\left.\mathrm{dN}_{\mathcal{C}} / \mathrm{dy}\right\rangle 20$ it could be interpreted as a signal for the quark-gluon plasma. This requires very careful data analysis because of the decreasing signal to background. The 40 fold increase in the number of events should permit us to answer this fascinating question.

b) Mass identified particle yields in $\bar{p}-p$ collision at $\sqrt{\mathrm{s}}=1.8 \mathrm{TeV}$. In $\overline{\mathrm{pp}}$ collisions at $\sqrt{\mathrm{s}}=1.8 \mathrm{TeV}$ the yields and the transverse momentum distributions of $\pi^{ \pm}, \mathrm{K}^{ \pm}, \mathrm{p}^{ \pm}$in the central region have been measured up to $\mathrm{dN}_{\mathrm{C}} / \mathrm{dh}=16$. We observe that the increase of $\left\langle\mathrm{P}_{t}\right\rangle$ with increasing $d N_{\mathcal{C}} / \mathrm{dn}$ is more significant for heavier particles than for lighter particles. This effect can be interpreted as a superposition of transverse flow of the centrally produced matter and the thermal motion of the emitted particles. This work has been submitted to Phys. Rev. Lett. 


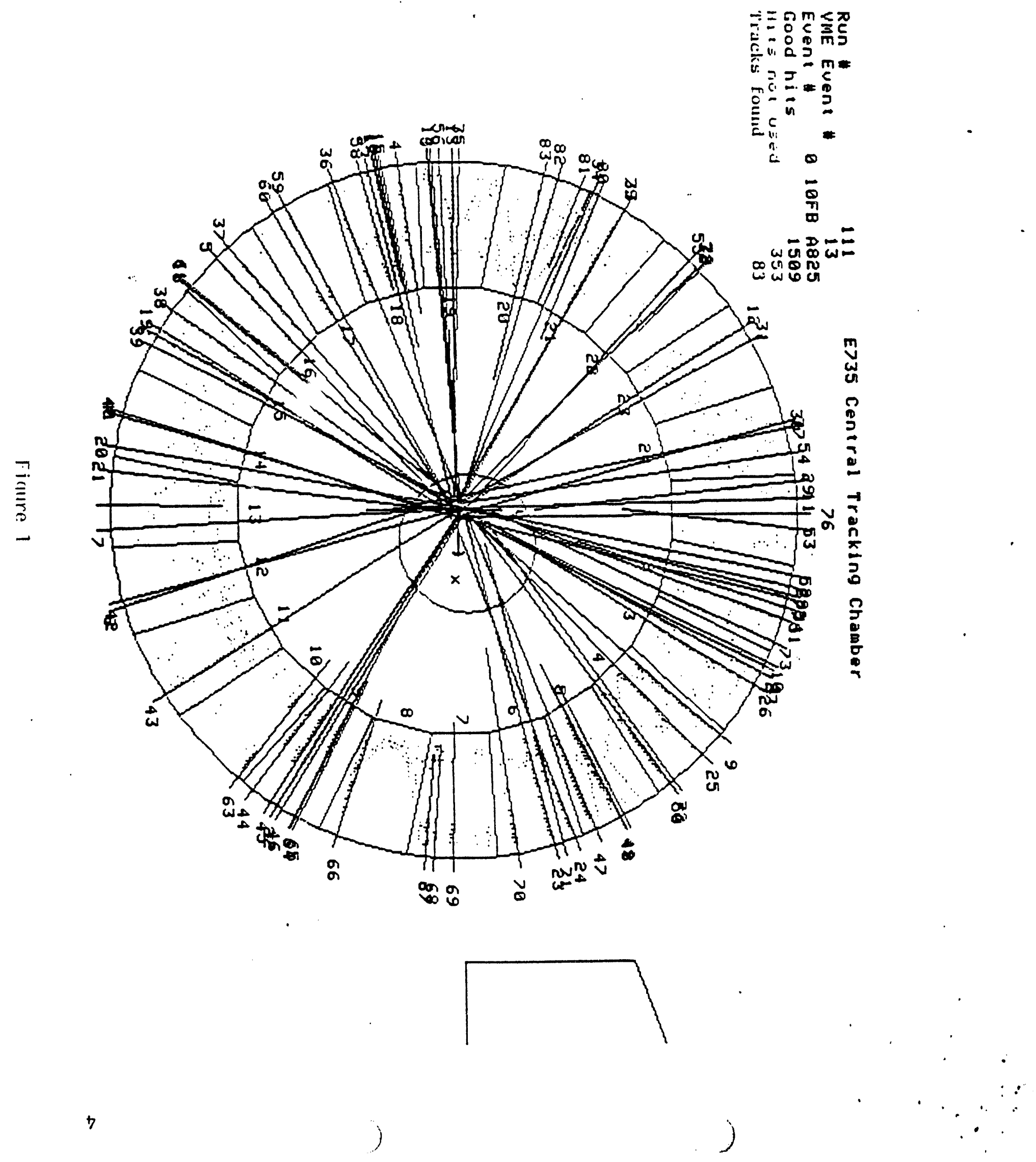



c) $\Lambda^{\circ}$ and $\Lambda^{\circ}$ Production from $\bar{p}-p$ collisions at $\sqrt{s}=1.8 \mathrm{TeV}$. We have measured the transverse momentum of the $\Lambda^{\circ} s$ and $\bar{\lambda}_{0}$ 's in pp collisions at $1.8 \mathrm{TeV}$. The average $\left\langle\mathrm{P}_{t}\right\rangle$ increases faster with center of mass energy, $\sqrt{\mathrm{s}}$ than that of the pions and the ratio of $\Lambda^{0}+\bar{\lambda}_{0}$ to all charge particles increases as a function of the charged particle multiplicity. We anticipate that the present run will produce many more $\Lambda^{0}+\bar{\Lambda}_{0}$ and allow a comparison with the $\bar{p}+p$ production as a function of the total charged particle multiplicity. This ratio of $\Lambda^{0}+\bar{\Lambda}_{0}$ to $\bar{p}+p$ could give information about the strange quark content in baryon production. These results from the 1987 test run have been published in Phys. Rev. Lett. and are appended to this document.

d) Energy dependence of $\bar{p}-p$ interactions at $\sqrt{s}=0.3 \mathrm{TeV} ; 1.0 \mathrm{TeV}$ and $1.8 \mathrm{TeV}$.

Analysis of the $\bar{p}-p$ collisions at three energies as a function of the charged particle multiplicity have been completed. This more detailed paper will be submitted to the Physical Review.

e) CTC design and evaluation.

A full description of the central tracking chamber design, construction and evaluation is under preparation. The chamber has state of the art two track resolution and a revolutionary low mass structure based on graphite-epoxy laminates. The electronics is a design by the University of Heidelberg produced by struck \& Co. Details of cross talk cancellation, charge division performance and noise isolation are discussed. This paper will be submitted to Nuclear Instruments and Methods. 
.IV. Exclusive nuclear fragmentation experiment at the Bevelac using the new TPC.

It is proposed to do an exclusive nuclear fragmentation experiment using a $1.6 \mathrm{GeV} /$ nuclear medium weight nucleus incident on a light target as shown below.

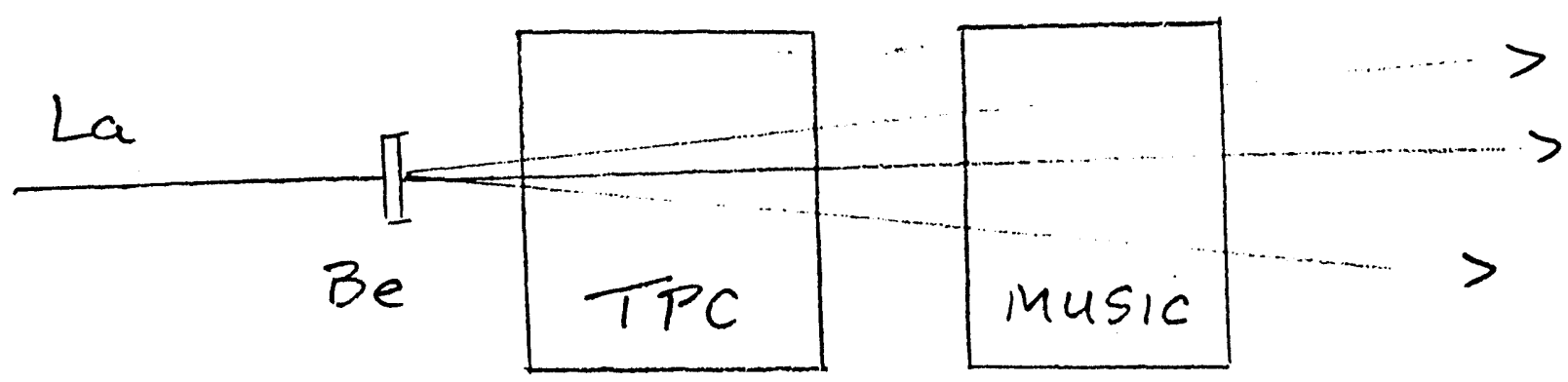

The reverse kinematics geometry assures that all fragments enter the time projection chamber (TPC) and or the multiple sampling ionization chamber (MUSIC). The TPC can handle $\mathrm{z}$ identification from 1 to 8 , MUSIC from 6 to the highest $z$ value. In this analysis all fragments are sorted out according to their atomic number. With this information it is possible to use a percolation theory model to measure the critical indices. The percolation model is scaled down to a system of $\sim 150$ nucleons. The Purdue group is responsible for event simulation and the design and .. construction of the trigger system. A letter of intent will be sent by the LBL-multi-university collaboration by the end of the year. A full proposal will be submitted to the PAC early in 1990.

It should be noted that these unique signals of a nuclear matter phase transition may be adapted to the quark-gluon phase transition and form the basis for a novel RHIC detector. We are interested in developing the planar detectors (PPAC) for such a detector.

V. Research and development on parallel plate avalanche chambers (PPAC) Recent measurements have shown the advantages of a mesh parallel plate geometry and the development of wireless multistage PPAC's. Multigap structures would allow gating of the detector which can thus be used in a 
high rate environment $>10^{7} / \mathrm{sec}$. Two dimensional readout with either light or charge signals can be used so that high multiplicity measurements would be possible. Mesh construction would make the building of large detectors practical. Such detectors coupled with a cathode pad readout could be a very useful tracking detector in the RHIC environment.

It is proposed to investigate the construction of a large area planar detector with emphasis on the gating feature and the associated electronics. We will be able in the initial phase to provide the means for a prototype unit from the existing contract funds. If this develops into a full sized counter, we will ask for RHIC development funds in the 1991 fiscal year.

\section{Data Analysis}

(a) Computer System

During the fall of 1988 we installed a Microvax 3600 server. In 1989 we made several significant additions to our computer system. An $8 \mathrm{~mm}$ tape drive has enabled us to begin analysis of data summary tapes produced from Fermilab experiment E-735. Approximately ten 9 track tapes worth of data can be stored on one $8 \mathrm{~mm}$ tape, greatly reducing the number of data summary tapes. We have also acquired a vaxstation 3100, thus doubling our cpu capabilities. The vaxstation is a satellite node with the Microvax as the boot node. The vaxstation has been used extensively in our simulation of E-735. It is ideal not only because it provides excellent graphic capabilities which are essential to a simulation program such as GEANT, but also because these simulations tend to be very cpu intensive. With this system we can efficiently work on our simulations and analyze data.

(b) Simulations

During the past year a major effort has been devoted to simulations for $E-735$. In order to understand the trigger system and central tracking chamber, we have developed a simulation based on GEANT. In addition, this has allowed us to study the effects of our off-line software. This knowledge is necessary for correcting the data for inefficiencies and bias in both the experimental apparatus and the 
analysis software. We have also developed a simulation of the spectrometer arm. This will be used to study the tracking algorithm used on spectrometer tracks, and to make acceptance corrections as a function of transverse momentum, particle type, and multiplicity. An example of the simulation studies we conducted is investigation into two distinct tracking algorithms for the CTC. The "phi filter" algorithm exploits the lack of a magnetic field in the CTC. Charged primaries from a beam-beam collision should have a constant phi as they traverse the CTC. Peaks in the phi distribution of the hits are clusters of correlated hits. Since the sense planes are tilted $5^{\circ}$ away from the beam position, the images of primary" tracks will not form a cluster. In the same way, secondaries and their images are not well seen since they will usually not point back to the beam line.

The "line search" algorithm is a more traditional iterative search procedure. It initially looks for short line segments formed by a few hits. A line is fit and then a search for hits along the line is made. This algorithm is not biased towards or against tracks originating from the beamline.

our simulation produces primary and secondary hits in the CTC which are then encoded into Flash ADC spectra (amplitude versus time). A hit detection algorithm is then applied, recovering the spatial information. Although this appears to be a circular exercise, an important feature which the simulation provides is a realistic fraction of tracks which overlap in time. Thus, the point of the study is to determine which of the two above algorithms is more . efficient in assigning hits to their correct tracks. Only via simulation can this question be answered.

Both algorithms produced similar results for fraction of primary tracks found, fraction of hits used in tracks, etc. The computation time was similar for the implementations. In the end, the line search was chosen, mainly since it can find tracks not pointing towards the beamline. 

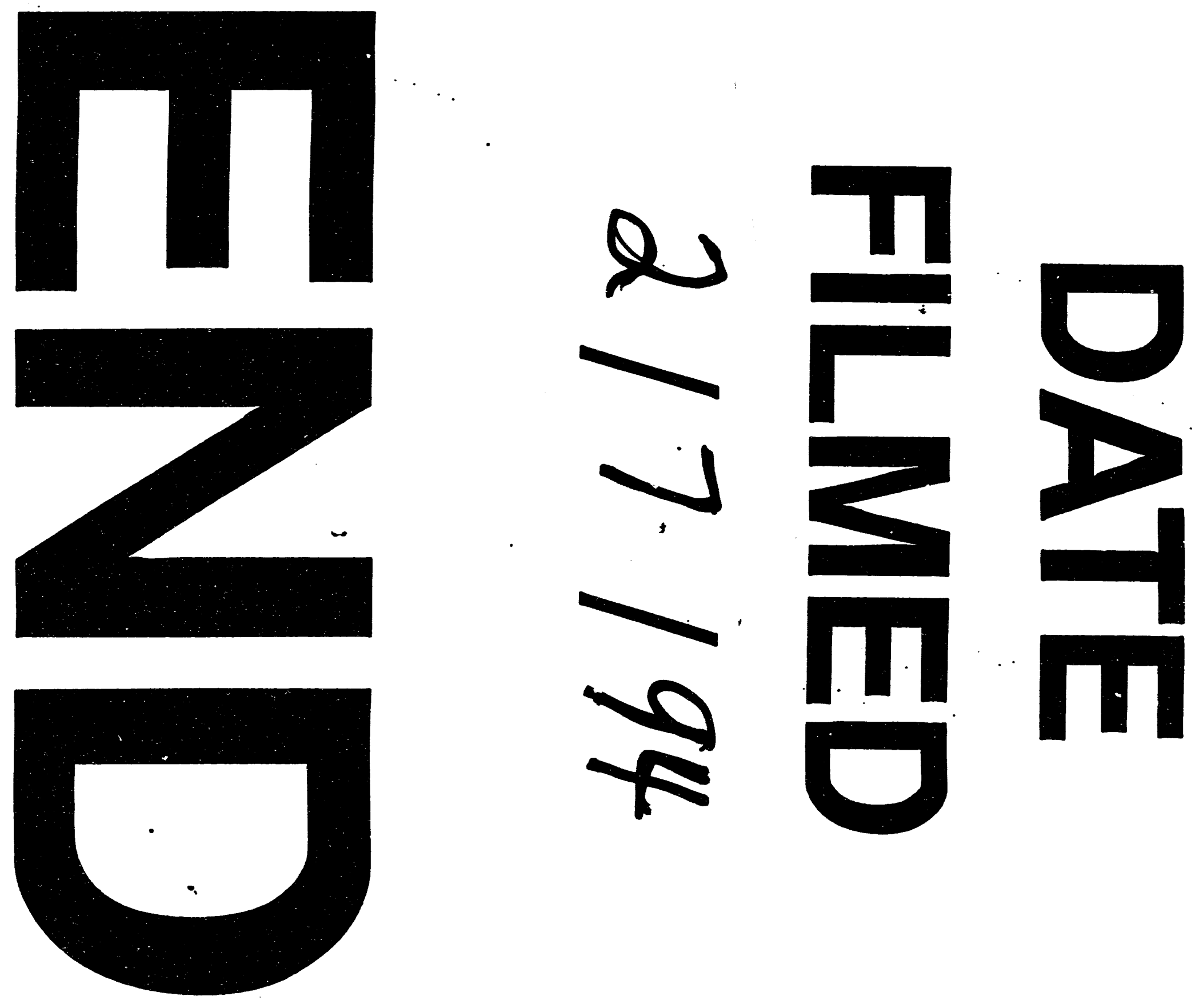
$$
\text { , }
$$ 